Государственное учреждение здравоохранения

«Тульский областной онкологический диспансер»
ЗНАЧЕНИЕ АДЪЮВАНТНОЙ

И ПАЛЛИАТИВНОЙ ПРОТИВООПУХОЛЕВОЙ

ЛЕКАРСТВЕННОЙ ТЕРАПИИ

ДЛЯ СНИЖЕНИЯ СМЕРТНОСТИ ОТ

ЗЛОКАЧЕСТВЕННЫХ НОВООБРАЗОВАНИЙ

E.В. Карабина

\section{THE VALUE OF ADJUVANT AND PALLIATIVE ANTITUMOR DRUG TREATMENT IN REDUCTION MORTALITY FROM MALIGNANT NEOPLASMS}

\author{
Е.В. Карабина \\ врач-онколог, \\ заведующая отделением противоопухолевой лекарственной терапии, \\ Тульский областной онкологчческий диспансер. \\ Россия, Тула, ул. Яблочкова, д. 1 Б, Стащионар 300053. \\ Тел.: 8 (4872) 48-62-81; 8(910) 55489 07; \\ E-mail: kev-251@yandex.ru. \\ E.V. Karabina \\ Oncologist, \\ Head of the Antitumour Drug Treatment Department, \\ Tula Regional Oncology Center. \\ Russia, Tula, ul. Yablochkova, 1 B, Hospital 300053. \\ Phone: 8 (4872) 48-62-81; 891055489 07; \\ E-mail: kev-251@yandex.ru.
}

Несмотря на значительное количество научных открытий в сфере онкологии, снижение показателей смертности от злокачественных новообразований по разным данным составило всего 10-20\%. Очень ограниченное количество заболеваний имеет доказанный суммарный выигрыш от использования профилактической химиотерапии и в меньшей степени от паллиативной противоопухолевой лекарственной терапии. Вклад этих методов лечения в снижение смертности от всех злокачественных новообразований теряется и не имеет точного цифрового выражения. Основная проблема отсутствия четких данных заключается в невозможности автоматически экстраполировать конечные статистические точки в клинических исследованиях на общую популяцию и приравнять некоторые из них к эпидемиологическому показателю смертности. С позиции доказательной медицины наиболее убедительно выглядит значение адъювантной и паллиативной лекарственной терапии для снижения смертности от рака молочной железы и колоректального рака. Уменьшение смертности от злокачественных новообразований представляет собой результат совокупного вклада экономических ресурсов систем здравоохранения, наличия скрининга и программ, направленных на устранение факторов риска развития 3НО, а также определенной инфраструктуры и средств для правильного проведения всех видов лечения $3 \mathrm{HO} \mathrm{(в} \mathrm{том} \mathrm{числе} \mathrm{реабилитационных} \mathrm{мероприятий),} \mathrm{уровня} \mathrm{образования}$ онкологов и их приверженности современным рекомендациям.

Ключевые слова: слертность от злокачественных новообразований, адъювантная терапия, паллиативная терапия, снижение смертности.

Despite the significant number of scientific discoveries in the field of cancer research, the reduction of mortality rate from malignant neoplasms according to various sources amounts to only 10-20\%. A very limited number of diseases boast the terminal payoff in using adjuvant chemotherapy and this effect is even less in palliative antitumor drug treatment. The contribution of these treatments in reducing mortality from 
all malignant neoplasms is lost and has no precise digital expression. The main problem of the lack of precise data is the inability to automatically extrapolate the statistical end points in clinical research studies on the general population and equate some of them to epidemiologic mortality rate. From the perspective of evidence-based medicine the significance of adjuvant and palliative drug treatment to reduce mortality from breast cancer and colorectal cancer looks most compelling. The decrease in mortality from malignant neoplasms is the result of cumulative contributions of the economic resources of health systems, availability of screening and programs aimed at addressing risk factors in the development of malignant tumors, as well as certain infrastructure and facilities for the proper conduct of all types of cancer treatment (including rehabilitation measures), level of education of oncologists and their adherence to current guidelines.

Keywords: mortality from malignant neoplasms, adjuvant treatment, palliative antitumor drug treatment, mortality rate reduction.

$\mathrm{O}$ ценка результативности мероприятий по борьбе с онкологическими заболеваниями на популяционном уровне становится все более важной задачей для большинства стан мира. Тенденции по показателю выживаемости, наряду с тенденциями заболеваемости и смертности на основе популяционных оценок обеспечивают ключевое понимание общей эффективности системы здравоохранения [1]. Анализ деятельности и состояния здравоохранения также невозможен без учета демографической ситуации, возрастно-половой, этнической структуры населения, анализа миграционных процессов и других демографических показателей [2].

К сожалению, несмотря на значительное количество научных открытий в сфере онкологии, внедрение и совершенствование скрининговых программ, непрерывное развитие фармацевтической промышленности, за минувшие десятилетия (с 1953 по 2010 гг.) снижение показателей смертности от злокачественных новообразований (3НО) по разным данным составило всего 10-20\%, тогда как в отношении других заболеваний с высокой летальностью этот показатель снизился более чем на 60-70\% [3].

На первый взгляд, абсолютно очевиден тот факт, что снижение показателя смертности от ЗНО зависит не только от программ скрининга, но и от адекватного применения адъювантной лекарственной терапии, сроков ее начала, наличия определенной инфраструктуры и средств для проведения профилактического лечения, уровня образования онкологов и экономических ресурсов систем здравоохранения. Не менее важным этапом на пути решения проблемы снижения смертности от $3 \mathrm{HO}$ представляется статистический анализ и понимание «вклада» определенного 3НО (включая его особые подтипы) в негативные событийные показатели как на уровне общей популяции, так и в зависимости от подгрупповых различий (пол, возраст).

Если посмотреть на общемировые показатели смертности от 3 НО у мужчин и у женщин (рис. 1), можно выделить наиболее значимые по своему удельному весу локализации для каждого пола в отдельности и, зная современные рекомендации по назначению адъювантной лекарственной терапии, убедиться в том, что только очень ограниченное количество заболеваний имеет доказанный выигрыш

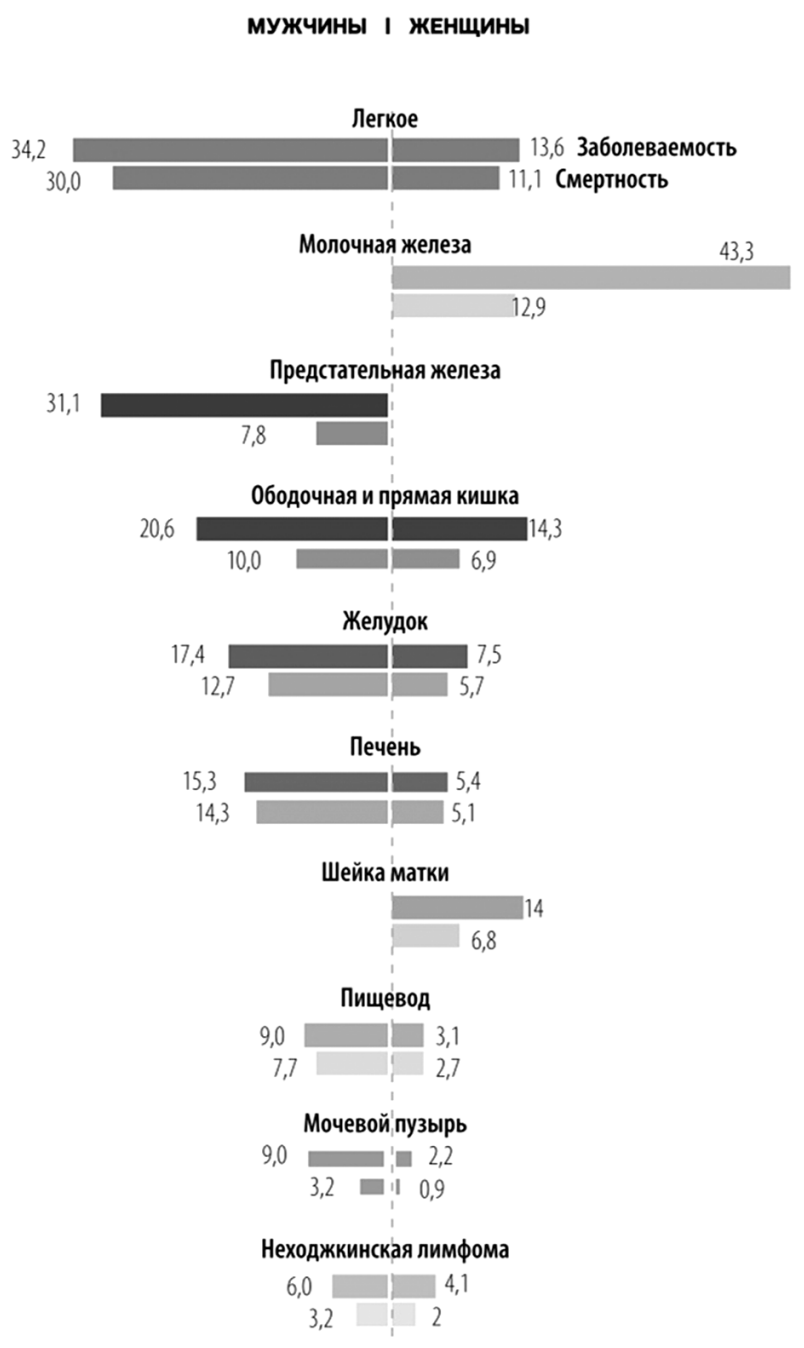

Рисунок 1. Приблизительная заболеваемость и слертность от рака во всем мире, стандартизованная по возрасту на 100 тыс. человек по локализацияп у мужсиин

и женщин, 2012 2. [4]

от использования профилактической химиотерапии. Поэтому влияние адъювантной противоопухолевой лекарственной терапии на снижение смертности от всех злокачественных новообразований в глобальном масштабе видится весьма неоднозначным. С позиции доказательной медицины и общемировой статистики довольно убедительно выглядит значение адъювант- 
ной лекарственной терапии для снижения смертности от рака молочной железы, поскольку данный вопрос является относительно изученным, и этой теме посвящено немалое количество публикаций. Кроме того, вышеуказанное заболевание остается ведущей причиной смерти среди всех причин онкологической смертности женщин во всем мире.

Из 184 стран, охватываемых базой данных GLOBOCAN, рак молочной железы является самым распространенным онкологическим диагнозом уженщин в 140 странах (76\%) и самой частой причиной смерти от рака в 101 стране (55\%) [4]. Несмотря на возрастающий уровень заболеваемости раком молочной железы, отмечается стойкое постоянное снижение смертности от данной патологии (в основном это касается стран с высоким доходом) [4]. Например, в США смертность от рака молочной железы за период 1990-2010 гг. уменьшилась с 33,0 до 21,3 на 100000 женского населения или на 36\% [5]. В европейской популяции (40 стран) в 2012 г. скорректированная по возрасту ежегодная частота возникновения (заболеваемость) раком молочной железы (РМЖ) достигла 94,2\%, а смертность - 23,1\% (IARC-EUCAN, 2015) [6]. При этом в большинстве западных стран в последние годы снижение показателей смертности от РМЖ, особенно в более молодых возрастных группах, происходит благодаря улучшению лечения и раннему выявлению заболевания [7, 8]. Sun Е. и соавторы, а также Toriola A.T. и соавторы заключают, что между 1988 и 2000 гг. улучшение выживаемости больных РМЖ в США в большей степени связано с улучшением лечения, чем с самим скринингом [9, 10].

Еще в 2005 г. Berry D.A. и соавторы проанализировали результаты рандомизированных исследований по скринингу рака молочной железы и пришли к выводу о том, что из 20-25\% снижения смертности маммографический скрининг ответственен только лишь за половину [11]. Вторая половина снижения смертности обеспечивается современной эффективной адъювантной гормоно- и химиотерапией, а также адъювантной лучевой терапией. Главный вывод из этого анализа заключался в потенциировании эффектов скрининга и лечения.

Интересен тот факт, что снижение смертности от рака молочной железы одновременно отмечается и у женщин, не участвующих в программе скрининга. При анализе снижения смертности в группе скрининга,по мнению рядя исследователей, на его долю приходится лишь одна треть $[12,13]$, в то время как современное лечение ответственно за остальные две трети снижения.

Таким образом, использование только скрининга без адъювантной терапии или адъювантной терапии без скрининга способно лишь стабилизировать смертность, но не уменышить ее. Недавний мета-анализ Оксфордской группы показал, что поли-химиотерапия, включающая антрациклины и таксаны, сокращает смертность от РМЖ (в популяции больных РМЖ) приблизительно на одну треть, в основном независимо от опухолевых характеристик (размер, состояние лимфоузлов, гистологический тип, степень злокачественности, гормонально-рецепторный статус) [14].

Национальный институт рака США (NCI) проанализировал влияние скрининга и лечения на снижение смертности от рака молочной железы [15]. Исследователи исходили из следующей гипотезы: если скрининг ответственен на 100\% за это снижение, тогда у больной, у которой диагноз поставлен в 1973-1979 гг. (когда еще не было адъювантной терапии), и у больной такого же возраста, с таким же размером опухоли, стадией и рецепторным статусом, диагностированным в 2005-2010 гг., риск смерти от рака молочной железы должен быть одинаковым. Предполагалось то, что если риск смерти будет различаться, то тогда за это различие ответственно современное лечение, основу которого составляет системная адъювантная терапия. Оказалось, что для женщин моложе 70 лет размер первичной опухоли, стадия заболевания (критерий, зависимый от скрининга) и рецепторный статус ответственны лишь за 17\% снижение смертности в течение первых 5 лет после постановки диагноза, в то время как лечение ответственно за 87\% снижения риска смертности. У больных старше 70 лет размер первичной опухоли, стадия и рецепторный статус играли большее значение в снижении смертности, они ответственны за 49\% снижения у больных с N0 и 38\% - у больных с наличием метастазов в регионарных лимфоузлах $(\mathrm{N}+)$. Таким образом, в популяции женщин, которым рекомендован скрининг (старше 50 лет и моложе 70 лет), основной причиной снижения риска смерти от рака молочной железы являются современные методы лечения, в частности, адъювантная лекарственная системная терапия.

К сожалению, такие выводы как «сокращение смертности от РМЖ после внедрения скрининговой маммографии было преимущественно результатом улучшенной системной терапии», основаны на субъективном видении авторов, и не имеют точного цифрового выражения («приблизительные расчеты») [16]. Основная проблема отсутствия четких данных заключается в невозможности автоматически әкстраполировать конечные статистические точки в клинических исследованиях на общую популяцию и приравнять некоторые из них к эпидемиологическому показателю смертности. Расчеты данного критерия в общей популяции вне рамок клинических исследований (mortality rate) осуществляют на среднегодовое население [2]. Принципиально важно разграничивать понятия смертности и летальности, так как последний показатель (case fatality rate) представляет собой отношение числа умерших от определенного заболевания к числу переболевших этой болезнью.

В клинических исследованиях очень сложно оценить влияние того или иного препарата на пока- 
затель смертности. Как правило, производится оценка снижения риска смерти. Однако и с этим критерием могут возникнуть объективные сложности, особенно если изучается эффективность какого-либо препарата в лечебном или паллиативном плане, так как пациент при непрерывно-прогрессирующем течении заболевания получает более 2 линий лекарственной терапии. Изучение показателя смертности потенциально может потребовать длительного периода наблюдения и с маркетинговой точки зрения не выгодно ни для промоции, ни для регистрации препарата.

Существует ограниченное количество исследований, в которых в качестве конечной или дополнительной точки выбирается показатель снижения риска смерти. Еще в 2005 г. Edward H. и Romond представили сводные результаты двух исследований по сравнению адъювантной химиотерапии с или без применения трастузумаба у пациенток с резектабельным HER2позитивным раком молочной железы [17]. Было показано, что добавление трастузумаба сокращало риск смерти на треть $(\mathrm{P}=0,015)$. Среди пациентов, которые продолжали лечение после доксорубицина и циклофосфамида и имели HER2-позитивный статус по результатам централизованного тестирования, относительное снижение риска смерти, связанного с трастузумабом составило $39 \%(\mathrm{P}=0,01)$ [17]. В целом, длительность клинических испытаний редко бывает достаточной, чтобы просмотреть за всеми пациентами до самой смерти. Снижение риска смерти в конкретном исследовании не эквивалентно снижению смертности от 3НО (в данном случае от рака молочной железы).

Для оценки показателей смертности от злокачественных новообразований необходимо наличие данных о смертности населения от всех причин смерти. Использование абсолютных чисел в анализе смертности не дает возможности правильно оценить уровень данного показателя и его динамику [2]. Статистические данные о случаях смерти в клинических исследованиях на хорошо отобранных группах больных, получаемые в результате текущего наблюдения, отражают абсолютные цифры, позволяя вычислить снижение риска смерти, но не дают возможности проецирования этих данных на общую популяцию. В общей популяции наиболее высоки коэффициенты смертности от злокачественных новообразований в самых старших группах. Следовательно, увеличение обычных коэффициентов смертности отражает не подлинный рост смертности от рака, а вызвано в основном изменением возрастного состава населения, точнее увеличением в населении доли лиц пожилого возраста, более склонного к поражению раком [2]. Кроме того, у пожилых пациентов значительно ограничены возможности для проведения адъювантной противоопухолевой лекарственной терапии, что косвенным образом может оказывать влияние более высокие показатели смертности в указанной группе.
Для того чтобы оценить абсолютный вклад адъювантной лекарственной терапии в снижение смертности от 3НО, необходимо четко понимать, что данный метод лечения назначается по строгим показаниям (а они могут меняться по мере появления новых результатов исследований и рекомендаций), в нужное время, в нужном объеме, нужным пациентам вне зависимости от социального статуса, после адекватно выполненного хирургического вмешательства, при адекватном стадировании и получении «нужных» морфологических и иммуногистохимических показателей.

Согласно результатам исследований, опубликованным в 2016 г. в журнале JAMA Oncology, показатель общей выживаемости больных раком молочной железы напрямую зависит от временного интервала между операцией и началом проведения адъювантной химиотерапии [18]. Похожие работы, посвященные проблемам клинических последствий затягивания начала адъювантной химиотерапии у пациенток с раком молочной железы встречались в более ранних публикациях и включали не более 6827 пациенток [19, 20]. Профессор Мариана Чавез МакГрегор совместно с коллегами из Онкологического центра Андерсона Техасского университета проанализировали результаты популяционного наблюдательного исследования, в котором приняли участие 24843 больных, и сделали вывод о том, что начало проведения адъювантной химиотерапии спустя 91 и более дней после выполнения операции снижает общую выживаемость больных РМЖ и выживаемость, зависящую непосредственно от заболевания. Кроме того, было продемонстрировано то, что каждое последующее увеличение временного интервала на 30 дней способствовало увеличению риска смерти, независимо от ее причины, на 9-10\% (ОР 1,10; 95\% ДИ 1,07-1,13; $\mathrm{p}<0,001$ ).

Авторы также показали, что начало проведения адъювантной химиотерапии спустя 91 и более дней от выполненной операции приводит к увеличению риска смерти на 34\% (ОР 1,34; 95\% ДИ 1,15-1,57), а риска смерти от РМЖ - на 27\% (ОР 1,27; $95 \%$ дИ 1,05-1,53). При проведении подгруппового анализа в зависимости от молекулярного подтипа РМЖ оказалось, что чем больше был временной интервал до начала проведения адъювантной химиотерапии, тем ниже были показатели общей выживаемости (ОР 1,53; $95 \%$ ДИ 1,17-2,00) и выживаемости, зависящей от заболевания (ОР 1,53;95\% ДИ 1,17-2,07) больных РМЖ с тройным негативным фенотипом [16]. Не менее важно своевременное начало профилактической химиотерапии при III стадии рака ободочной кишки, так как отсрочка ее начала на каждые 4 недели приводит к повышению риска смерти на 14\%, а при сроке свыше 3 месяцев выигрыш от проведения данного лечения сводится к минимуму [21].

При анализе информации, представленной в медицинской базе данных SEER и регистре онколо- 
гических заболеваний в Калифорнии, выявлены ряд факторов, ставших причинами несвоевременного проведения лечения. Основными из них были латиноамериканское происхождение и негроидная раса больных [22]. В большинстве ранее проведенных исследований именно у представителей малых этнических групп и у пациенток, имеющих низкий социально-экономический статус, чаще всего наблюдалось несвоевременное проведение лечения. Длительные временные интервалы могут быть одной из причин того, что в группах «непривилегированных» больных РМЖ показатели выживаемости ниже по сравнению с другими пациентами [22].

B 2016 г. в журнале THE LANCET была опубликована статья, посвященная влиянию глобального экономического кризиса на смертность от рака. Maruthappu M. с соавторами выполнили оценку влияния изменения уровня безработицы и расходов государственного сектора на здравоохранение (РГЗ) на смертность от рака, и установлено, какое влияние всеобщий охват медицинским обслуживанием (ВОМО) оказывает на эту взаимосвязь [23]. Авторы исходили из того, что глобальный экономический кризис сопровождается ростом безработицы и снижением РГЗ. При проведении продольного анализа, были получены данные от Всемирного банка и ВОЗ (1990-2010 гг.) и объединены показатели смертности от рака молочной железы у женщин, рака предстательной железы у мужчин и колоректального рака у мужчин и женщин, которые характеризовались выживаемостью более $50 \%$, в класс онкологических заболеваний, поддающихся лечению. Подобным же образом исследователи объединили данные для рака легких и рака поджелудочной железы, которые характеризуются 5-летней выживаемостью менее $10 \%$, в класс неизлечимых онкологических заболеваний. Для изучения взаимосвязи между безработицей, РГЗ и смертностью от рака, с учётом и без учета ВОМО, авторами использован мультипараметрический регрессионный анализ с контролем демографических параметров и инфраструктуры конкретных стран, анализ интервала запаздывания и проверки надежности. Также был применен анализ тенденций для прогнозирования показателей смертности на основании тенденций, наблюдавшихся до резкого роста безработицы, отмечавшегося во многих странах с 2008 по 2010 гг., и проведено сравнение их с наблюдаемыми показателями.

Для анализа безработицы были доступны данные из 75 стран с населением 2,106 млрд человек, а для анализа РГЗ - данные из 79 стран с населением 2,156 млрд человек. Выявлена значимая взаимосвязь роста безработицы с повышением смертности от всех видов рака и смертности от конкретных видов рака, за исключением рака легких у женщин. В противоположность этому, для смертности от неизлечимых видов рака не было выявлено значимой взаимосвязи с изменением уровня безработицы. Анализ интер- вала запаздывания показал сохранение значимой взаимосвязи на протяжении 5 лет после повышения безработицы для класса излечимых видов рака. При повторном анализе с учетом статуса ВОМО значимая взаимосвязь исчезала. Повышение РГЗ сопровождалось значимым снижением смертности от всех видов рака, поддающихся лечению, и смертности от конкретных видов рака. При проведении анализа временных рядов для 2000-2007 гг. получено более чем 40000 дополнительных случаев смерти от множества поддающихся лечению видов рака с 2008 по 2010 гг. Большинство из этих смертей приходились на страны без ВОМО. На основании сложных анализов авторы сделали вывод о том, что повышение безработицы связано с увеличением смертности от рака и ВОМО, по-видимому, нивелировало этот эффект. Также было показано, что повышение РГЗ связано со снижением смертности от рака. Данная взаимосвязь может объясняться доступом к медицинской помощи. По оценкам исследователей, только в странах Организации экономического сотрудничества и развития экономический кризис 2008-2010 гг. привел к 260000 дополнительных случаев смерти от рака [23].

В условиях нестабильной экономики возникает необходимость не только в формировании адекватного канцер-регистра и определения наиболее значимых нозологий для оптимизации распределения финансирования лекарственного обеспечения, но и соответствие существующих стандартов адъювантной терапии эффективной клинической практике, возможность обеспечения достойного поддерживающего лечения и реабилитационных мероприятий, минимизация «финансовой токсичности» лечебного процесса, сохранение запланированной дозы цитостатиков, приверженность к выполнению клинически одобренных рекомендаций. Адъювантная лекарственная терапия в качестве изолированной опции вряд ли обеспечит абсолютное снижение смертности от рака молочной железы. В исследовании Рan Н. и соавторов было показано, что при эстроген-зависимом раке молочной железы смертность от этого заболевания на 34\% выше у больных с ожирением по сравнению с пациентками без ожирения [24]. Контроль массы тела у пациенток раком молочной железы - эффективный метод снижения смертности после проведенного противоопухолевого лечения.

Обсуждая вопросы влияния адъювантной лекарственной терапии на снижение смертности от 3НО, хочется акцентировать внимание на том, что данное лечение само по себе не должно вызывать серьезные нежелательные явления, сопряженные с высоким риском летальных исходов и тем более, смерть на фоне указанной терапии. В недавно опубликованном исследовании II фазы по применению ипилимумаба в адъювантной терапии у пациентов с III стадией меланомы было показано, что нежелательные явления 3 и 4 степени в группе больных, получавших ипилимумаб, 
встречались в 54,1\%, а иммуно-опосредованные нежелательные явления 3 и 4степеней - в 41,6\% случаев. 5 из 475 пациентов в группе ипилимумаба умерли от иммуно-опосредованных нежелательных явлений $(1,1 \%): 3$ от колита (2 пациента с интестинальной перфорацией), 1 пациент от миокардита и 1 пациент от полиорганной недостаточности, ассоциированной с синдромом Guillian-Barre [25]. При этом 5-летняя безрецидивная выживаемость в группе ипилимумаба была 40,8\%, а в группе контроля 30,3\% (ОР 0,76; 95,8 [CI], 0,64-0,89; P<0,001); показатель общей 5-летней выживаемости составлял 65,4\% в группе ипилимумаба и $54,4 \%$ в группе плацебо (ОР 0,$72 ; 95,1 \%$ CI, $0,58-0,88$; $\mathrm{P}=0,001)$ [25]. Не слишком ли дорогая цена за разницу в показателях 10\%? Смысл адъювантной терапии теряется, если она сопряжена с фатальными событиями и высоким риском жизне-угрожающих осложнений.

Имеющиеся исследования по анализу влияния адъювантной химиотерапии на снижение смертности от ЗНО демонстрируют приблизительные значения сокращения данного показателя от профилактического использования противоопухолевой лекарственной терапии при ограниченном числе нозологий (рак молочной железы у женщин, колоректальный рак у мужчин и женщин, в некоторых странах рак желудка). В глобальном масштабе вклад данного влияния на снижение смертности от всех 3 НО теряется и на первый взгляд не меняет ситуацию существенным образом. С позиции доказательной медицины и общемировой статистики довольно убедительно выглядит значение адъювантной лекарственной терапии для снижения смертности от рака молочной железы и колоректального рака. Констатация наличия әффективной комбинации, которая увеличивает продолжительность жизни пациентов до 5 лет - недостаточный шаг в достижении цели. Снижение показателей смертности от вышеуказанных 3 НО зависит не только от адекватного применения адъювантной лекарственной терапии, сроков ее начала, наличия определенной инфраструктуры, средств для проведения профилактического лечения, уровня образования онкологов, экономических ресурсов систем здравоохранения, единых рекомендаций, но и от скрининга и программ, направленных на устранение факторов риска развития $3 \mathrm{HO}$.

Поиск путей улучшения результатов лечения онкологических больных (в особенности с метастатическими формами) и снижения смертности от $3 \mathrm{HO}$ остается очень сложной задачей. Вклад отдельных цитостатиков и ряда других противоопухолевых препаратов в выживаемость больных распространенным опухолевым процессом нельзя назвать драматическим в отношении абсолютного выигрыша и прибавки (зачастую медиана выживаемости меняется от нескольких дней [26] до нескольких месяцев). Безусловно, группа пациентов, которая получает противоопухолевую лекарственную терапию, по поводу метастатического заболевания с паллиативной целью весьма неоднородна. «Благодарная» таргетная популяция курабельных пациентов весьма ограничена (НМРЛ с активирующими мутациями, гормон-позитивный PMЖ, HER-позитивный РМЖ, GIST, рак почки, колоректальный рак, меланома с наличием активирующих мутаций и нормальным уровнем лактатдегирогеназы). Бурное развитие фармацевтической промышленности и обилие новых лекарственных препаратов формирует систему, которая заставляет делать выбор в пользу активного лечения больныхс распространенным опухолевым процессом. И врачи, и пациенты нередко становятся заложниками этой системы, не понимая, что качество жизни в некоторых ситуациях важнее ее количества. Погоня за прибавками жизни в несколько месяцев с сомнительным качеством - тренд, навеянный временем и «маркетинговыми оправданиями» фармацевтических компаний. «Сделайте все возможное» не тоже самое, что «сделайте все, что целесообразно». В жизни чаще всего медицинское сообщество слышит первую часть вышенаписанного предложения, тогда как вторая составляющая только подразумевается. «Сделайте все возможное» имеет более сильную эмоциональную окраску, которая отсутствует у выражения "сделайте все, что целесообразно». Особенно сложно действовать в ситуациях, когда для лечения распространенного онкологического заболевания существуют определенные рекомендации (вплоть до терминальных стадий) с указанием препаратов, от применения которых в $\mathrm{N}$-ной линии выигрыш более чем сомнителен. Современные тенденции расширения показаний при регистрации того или иного лекарственного препарата, особенно в ситуациях, когда при подгрупповых анализах были получены группы больных, отвечающих на лечение наилучшим образом, чреваты закреплением негативного опыта у медицинских работников и их пациентов. В широком понимании улучшение индивидуального прогноза при использовании лекарственной терапии метастатического процесса не определяет снижение смертности от 3НО. Даже если экстраполировать успехи лечения единичных больных на всю популяцию пациентов, страдающих распространенными 3НО, и представить идеальную ситуацию покрытия необходимыми препаратами всех нуждающихся, вряд ли удастся добиться снижения смертности.

При обзоре литературы не найдено ни одного исследования о влиянии паллиативной противоопухолевой лекарственной терапии на снижение смертности от ЗНО. Бесспорно, существующая «благодарная» таргетная популяция курабельных пациентов с метастатическим поражением (НМРЛ с активирующими мутациями, гормонопозитивный PMЖ, HERпозитивный PMЖ, GIST, рак почки, коло-ректальный рак, меланома с наличием активирующих мутаций и нормальным уровнем лактатдегирогеназы), предо- 
пределяет абсолютные и относительные успехи в лечении вышеуказанных заболеваний, но теряется в общей популяции больных и в глобальном масштабе не влияет на снижение смертности от 3НО. Означает ли это, что пациентов, страдающих распространенными формами ЗНО, не нужно лечить? Нет. Ответ абсолютно очевиден, поскольку индивидуальный, взвешенный и рациональный подход к каждому пациенту с метастатическим опухолевым процессом позволит обеспечить формирование оптимальной лечебной стратегии, не подвергая тяжелых больных «бесполезному лечению». В оправданных случаях попытка «хронизации» заболевания за счет последовательного использования имеющихся опций обеспечивает шансами на продление жизни. Паллиативную противоопухолевую лекарственную терапию следует рассматривать в качестве небольшой составляющей, вносящей вклад в суммарное увеличение продолжительности жизни больных 3 НО.

Обращаясь повторно к общемировым показателям смертности от $3 \mathrm{HO}$ у мужчин и у женщин (рис. 1), можно выделить наиболее значимые по своему удельному весу локализации для каждого пола в отдельности и, зная современные рекомендации по назначению адъювантной и паллиативной лекарственной терапии, убедиться в том, что только очень ограниченное количество заболеваний имеет доказанный суммарный выигрыш от использования профилактической химиотерапии и в меньшей степени от паллиативной противоопухолевой лекарственной терапии. Вклад этих методов лечения в снижение смертности от всех 3 НО теряется и не имеет точного цифрового выражения. С позиции доказательной медицины наиболее убедительно выглядит значение адъювантной и паллиативной лекарственной терапии для снижения смертности от рака молочной железы и колоректального рака. Констатация увеличения медианы общей и безрецидивной выживаемости не является основным фактором, определяющим снижение смертности. Уменьшение этого важного статистического показателя представляет собой результат совокупного вклада экономических ресурсов систем здравоохранения, наличия скрининга и программ, направленных на устранение факторов риска развития 3НО, а также определенной инфраструктуры и средств для правильного проведения всех видов лечения $3 \mathrm{HO}$ (в том числе реабилитационных мероприятий), уровня образования онкологов и их приверженности современным рекомендациям.

\section{Список литературы}

1. Quaresma M., Coleman M.P., Rachet B. 40-year trends in an index of survival for all cancers combined and survival adjusted for age and sex for each cancer in England and Wales, 1971-2011: a population-based study // Lancet. - 2015. - Vol. 385, № 9974. - P. 1206-18.

2. Merabishvili V.M. Oncological Statistics (traditional methods, new information technologies).Guidelines for physicians. Second edition, supplemented. Part I. - SPb. - 2015. - 223 p.

3. Решетник Д.А. Выжить любой ценой? Экономическое бремя лекарственной терапии в онкологии. // Газета российского общества клинической онкологии. - 2015. - № 1. С. 14-15.

4.Джемал А., Винеис П., Брей Ф., ТорреЛ., Форман Д. (редакция) Атлас по онкологии. Издание второе. Атланта, штат Джорджия: Американское онкологическое общество. - 2014. [Электронный ресурc] - URL: www.cancer. org/canceratlas (дата обращения 25.10.2016).

5. Narod S.A., Iqbal J., Miller A. Why have breast cancer mortality rates declined? // J. Cancer Policy. - 2015. Vol. 5. - P. 8-17.

6. International Agency for Research on Cancer (IARC). [Электронный pecypc] - URL: www.cancer.org/canceratlas (дата обращения 25.10.2016)..

7. Autier P., Boniol M., La Vecchia C., Vatten L., Gavin A., Héry C., Heanue M. Disparities in breast cancer mortality trends between 30 European countries: retrospective trend analysis of WHO mortality database // BMJ. - 2010. Vol. 341. - P. c3620.

8. Allemani C., Minicozzi P., Berrino F., Bastiaannet E., Gavin A., Galceran J., Ameijide A., Siesling S., Mangone L., Ardanaz E., Hédelin G., Mateos A., Micheli A., Sant M.; EUROCARE Working Group. Predictions of survival up to 10 years after diagnosis for European women with breast cancer in 2000-2002 // Int J Cancer. - 2013. - Vol. 132, № 10. P. 2404-12.

9. Sun E., Jena A.B., Lakdawalle D., et al. The contributions of improved therapy and earlier detection to cancer survival gains 1988-2000 // Forum Health Econ Policy. - 2010. - Vol. 132. - P. 1195.

10. Toriola A.T., Colditz G.A. Trends in breast cancer incidence and mortality in the United States: implications for prevention // Breast Cancer Res Treat. - 2013. - Vol. 138, №3. - P. 665-73.

11. Berry D.A., Cronin K.A., Pleuritis S.K., Fryback D.G., Clarke L., Zelen M., Mandelblatt J.S., Yakovlev A.Y., Habbema J.D., Feuer E.J.; Cancer Intervention and Surveillance Modeling Network (CISNET) Collaborators. Effect of screening and adjuvant therapy on mortality from breast cancer // N Engl J Med. - 2005. - Vol. 353, № 17. - P. 1784-92.

12.Lauby-Secretan B., Scoccianti C., Loomis D., Benbrabim-Tallaa L., Bouvard V., Bianchini F., StraifK.; International Agency for Research on Cancer Handbook Working Group. Breast-cancer screening--viewpoint of the IARC Working Group // Engl J Med. - 2015. - Vol. 372, № 24. - P. 2353-8. 
13.Kalager M., Zelen M., Langmark F., Adami H.O. Effect of screening mammography on breast-cancer mortality in Norway // N Engl J Med. - 2010. - Vol. 363, № 13. - P. 1203-10.

14. Early Breast Cancer Trialists' Collaborative Group (EBCTCG), Peto R., Davies C., Godwin J., Gray R., Pan H.C., Clarke M., Cutter D., Darby S., McGale P., Taylor C., Wang Y.C., Bergh J., Di Leo A., Albain K., Swain S., Piccart M., Pritchard K. Comparisons between different polychemotherapy regimens for early breast cancer: meta-analyses of long-term outcome among 100,000 women in 123 randomised trials // Lancet. - 2012. - Vol. 379, № 9814. - P. 432-44.

15. Park J.H., Anderson W.F., Gail M.H. Improvements in US Breast Cancer Survival and Proportion Explained by Tumor Size and Estrogen-Receptor Status // J Clin Oncol. - 2015. - Vol. 33, № 26. - P. 2870-6.

16. Welch H.G., Prorok P.C., O'Malley A.J., Kramer B.S. Breast-Cancer Tumor Size, Overdiagnosis, and Mammography Screening Effectiveness // N Engl J Med. - 2016. - Vol. 375, № 15. - P. 1438-1447.

17. Romond E.H., Perez E.A., Bryant J., Suman V.J., Geyer C.E. Jr, Davidson N.E., Tan-Chiu E., Martino S., Paik S., Kaufman P.A., Swain S.M., Pisansky T.M., Fehrenbacher L., Kutteh L.A., Vogel V.G., Visscher D.W., Yothers G., Jenkins R.B., Brown A.M., Dakhil S.R., Mamounas E.P., Lingle W.L., Klein P.M., Ingle J.N., Wolmark N. Trastuzumab plus adjuvant chemotherapy for operable HER2-positive breast cancer // N Engl J Med. - 2005. - Vol. 353, № 16. - P. 1673-84.

18. Chavez-MacGregorM., Clarke C.A., Lichtensztajn D.Y., Giordano S.H. Delayed Initiation of Adjuvant Chemotherapy Among Patients With Breast Cancer // JAMA Oncol. - 2016. - Vol. 2, № 3. - P. 322-9.

19. GagliatoDde. M., Gonzalez-Angulo A.M., Lei X., Theriault R.L., Giordano S.H., Valero V., Hortobagyi G.N., ChavezMacgregor $M$. Clinical impact of delaying initiation of adjuvant chemotherapy in patients with breast cancer // J Clin Oncol. - 2014. - Vol. 32, № 8. - P. 735-44.

20. Farolfi A., Scarpi E., Rocca A., Mangia A., Biglia N., Gianni L., Tienghi A., Valerio M.R., Gasparini G., Amaducci L., Faedi M., Baldini E., Rubagotti A., Maltoni R., Paradiso A., Amadori D. Time to initiation of adjuvant chemotherapy in patients with rapidly proliferating early breast cancer // Eur J Cancer. - 2015. - Vol. 51, № 14. - P. 1874-81.

21. Практические рекомендации по лекарственному лечению злокачественных опухолей / под редакцией: В.М. Моисеенко. - М. Общероссийская общественная организация Российское общество клинической онкологии. - 2015. - 456 с.

22. Общая выживаемость больных раком молочной железы: время имеет значение. Специализированное издание для онкологов под редакцией Русакова И.Г. Онкология сегодня. - 2016. - № 3(16). - С. 3.

23. Maruthappu M., Watkins J., Noor A.M., Williams C., Ali R., Sullivan R., Zeltner T., Atun R. Economic downturns, universal health coverage, and cancer mortality in high-income and middle-income countries, 1990-2010: a longitudinal analysis // Lancet. - 2016. - Vol. 388, № 10045. - P. 684-95.

24. Pan H., Gray R.G., on behalf of the Early Breast Cancer Trialist's Collaborative Group: Effect of obesity in premenopausal ER + early breast cancer: EBCTCG data on 80, 0000 patients in 70 trials. ASCO Annual Meeting. Abstract 503. Presented May 31, 2014.

25. Eggermont A.M., Chiarion-Sileni V., Grob J.J., Dummer R., Wolchok J.D., Schmidt H., Hamid O., Robert C., Ascierto P.A., Richards J.M., Lebbé C., Ferraresi V., Smylie M., Weber J.S., Maio M., Bastholt L., Mortier L., Thomas L., Tabir S., Hauschild A., Hassel J.C., Hodi F.S., Taitt C., de Pril V., de Schaetzen G., Suciu S., Testori A. Prolonged Survival in Stage III Melanoma with Ipilimumab Adjuvant Therapy // N Engl J Med. - 2016. [Epub ahead of print].

26. Moore M.J., Goldstein D., Hamm J., Figer A., Hecht J.R., Gallinger S., Au H.J., Murawa P., Walde D., Wolff R.A., Campos D., Lim R., Ding K., Clark G., Voskoglou-Nomikos T., Ptasynski M., Parulekar W.; National Cancer Institute of Canada Clinical Trials Group. Erlotinib plus gemcitabine compared with gemcitabine alone in patients with advanced pancreatic cancer: a phase III trial of the National Cancer Institute of Canada Clinical Trials Group // J Clin Oncol. 2007. - Vol. 25, № 15. P. 1960-6.

\section{References}

1.Quaresma M., Coleman M.P., Rachet B. 40-year trends in an index of survival for all cancers combined and survival adjusted for age and sex for each cancer in England and Wales, 1971-2011: a population-based study. Lancet. 2015 Mar 28; 385(9974): 1206-18. doi: 10.1016/S0140-6736(14)61396-9. PMID: 25479696.

2. Merabishvili V.M. Oncological Statistics (traditional methods, new information technologies).Guidelines for physicians.Second edition, supplemented. Part I. - SPb. 2015; 223 p.

3. Reshetnik D.A. Survive at any cost? The economic burden of drug therapy in oncology. // Newspaper of Russian Society of Clinical Oncology. 2015; 1:14-15. (In Russ).

4. Dzhemal A., Vineis P., Brej F., Torre L., Forman D. (editors) The cancer atlas. Available at: www.cancer.org/ canceratlas (accessed 25.10.2016).

5. Narod S.A., Iqbal J., Miller A. Why have breast cancer mortality rates declined? J. Cancer Policy. 2015; 5: 8-17.

6. International Agency for Research on Cancer (IARC). Available at: http://eco.iarc.fr/EUCAN/.aspx? Cancer $=24 \& G$ Gender $=2 \#$ blok-table-f (accessed 10.10.2016).

7. Autier P., Boniol M., La Vecchia C., Vatten L., Gavin A., Héry C., Heanue M. Disparities in breast cancer mortality trends between 30 European countries: retrospective trend analysis of WHO mortality database. BMJ. 2010 Aug 11; 341: c3620. doi: 10.1136/bmj.c3620. PMID: 20702548.

8. Allemani C., Minicozzi P., Berrino F., Bastiaannet E., Gavin A., Galceran J., Ameijide A., Siesling S., Mangone L., Ardanaz E., Hédelin G., Mateos A., Micheli A., Sant M.; EUROCARE Working Group. Predictions of survival up to 10 years 
after diagnosis for European women with breast cancer in 2000-2002. Int J Cancer. 2013 May 15; 132(10): 2404-12. doi: $10.1002 /$ ijc. 27895 .

9. Sun E., Jena A.B., Lakdawalle D., et al. The contributions of improved therapy and earlier detection to cancer survival gains 1988-2000.Forum Health Econ Policy. 2010; 132: 1195.

10. Toriola A.T., Colditz G.A. Trends in breast cancer incidence and mortality in the United States: implications for prevention. Breast Cancer Res Treat. 2013 Apr; 138(3): 665-73. doi: 10.1007/s10549-013-2500-7. PMID: 23546552.

11. Berry D.A., Cronin K.A., Plevritis S.K., Fryback D.G., Clarke L., Zelen M., Mandelblatt J.S., Yakovlev A.Y., Habbema J.D., Feuer E.J.; Cancer Intervention and Surveillance Modeling Network (CISNET) Collaborators. Effect of screening and adjuvant therapy on mortality from breast cancer. N Engl J Med. 2005 Oct 27; 353(17): 1784-92. doi: 10.1056/NEJMoa050518. PMID: 16251534.

12.Lauby-Secretan B., Scoccianti C., Loomis D., Benbrabim-Tallaa L., Bouvard V., Bianchini F., StraifK.; International Agency for Research on Cancer Handbook Working Group. Breast-cancer screening - viewpoint of the IARC Working Group. Engl J Med. 2015 Jun 11; 372(24): 2353-8. doi: 10.1056/NEJMsr1504363. PMID: 26039523.

13. Kalager M., Zelen M., Langmark F., Adami H.O. Effect of screening mammography on breast-cancer mortality in Norway. N Engl J Med. 2010 Sep 23; 363(13): 1203-10. doi: 10.1056/NEJMoa1000727. PMID: 20860502.

14. Early Breast Cancer Trialists' Collaborative Group (EBCTCG), Peto R., Davies C., Godwin J., Gray R., Pan H.C., Clarke M., Cutter D., Darby S., McGale P., Taylor C., Wang Y.C., Bergh J., Di Leo A., Albain K., Swain S., Piccart M., Pritchard $K$. Comparisons between different polychemotherapy regimens for early breast cancer: meta-analyses of long-term outcome among 100,000 women in 123 randomised trials. Lancet. 2012 Feb 4; 379(9814): 432-44. doi: 10.1016/S0140-6736(11)61625-5.

15. Park J.H., Anderson W.F., Gail M.H. Improvements in US Breast Cancer Survival and Proportion Explained by Tumor Size and Estrogen-Receptor Status. J Clin Oncol. 2015 Sep 10;33(26):2870-6. doi: 10.1200/JCO.2014.59.9191. PMID: 26195709.

16. Welch H.G., Prorok P.C., O'Malley A.J., Kramer B.S. Breast-Cancer Tumor Size, Overdiagnosis, and Mammography Screening Effectiveness. N Engl J Med. 2016 Oct 13; 375(15): 1438-1447. doi: 10.1056/NEJMoa1600249. PMID: 27732805.

17. Romond E.H., Perez E.A., Bryant J., Suman V.J., Geyer C.E. Jr, Davidson N.E., Tan-Chiu E., Martino S., Paik S., Kaufman P.A., Swain S.M., Pisansky T.M., Fehrenbacher L., Kutteh L.A., Vogel V.G., Visscher D.W., Yothers G., Jenkins R.B., Brown A.M., Dakhil S.R., Mamounas E.P., Lingle W.L., Klein P.M., Ingle J.N., Wolmark N. Trastuzumab plus adjuvant chemotherapy for operable HER2-positive breast cancer. N Engl J Med. 2005 Oct 20; 353(16): 1673-84. doi: 10.1056/ NEJMoa052122. PMID: 16236738.

18. Chavez-MacGregor M., Clarke C.A., Lichtensztajn D.Y., Giordano S.H. Delayed Initiation of Adjuvant Chemotherapy Among Patients With Breast Cancer.JAMA Oncol. 2016 Mar; 2(3):322-9. doi: 10.1001/jamaoncol.2015.3856. PMID: 26659132.

19. GagliatoDde. M., Gonzalez-Angulo A.M., Lei X., Theriault R.L., Giordano S.H., Valero V., Hortobagyi G.N., ChavezMacgregor M. Clinical impact of delaying initiation of adjuvant chemotherapy in patients with breast cancer. J Clin Oncol. 2014 Mar 10; 32(8): 735-44. doi: 10.1200/JCO.2013.49.7693. PMID: 24470007.

20. Farolfi A., Scarpi E., Rocca A., Mangia A., Biglia N., Gianni L., Tienghi A., Valerio M.R., Gasparini G., Amaducci L., Faedi M., Baldini E., Rubagotti A., Maltoni R., Paradiso A., Amadori D. Time to initiation of adjuvant chemotherapy in patients with rapidly proliferating early breast cancer. EurJCancer. 2015 Sep; 51(14): 1874-81. doi: 10.1016/j.ejca.2015.07.003. PMID: 26206258.

21. Practical recommendations on the drug treatment of malignant tumors / Edited by: Moiseenko V.M. - Moscow, Russian public organization Russian Society of Clinical Oncology. 2015. 456. (In Russ).

22. Overall survival of patients with breast cancer: time matters. Specialized edition for oncologists edited Rusakova I.G. / Oncology today. 2016; 3(16): 3. (In Russ).

23. Maruthappu M., Watkins J., Noor A.M., Williams C., Ali R., Sullivan R., Zeltner T., Atun R. Economic downturns, universal health coverage, and cancer mortality in high-income and middle-income countries, 1990-2010: a longitudinal analysis. Lancet. 2016 Aug 13; 388(10045): 684-95. doi: 10.1016/S0140-6736(16)00577-8. PMID: 27236345.

24. Pan H., Gray R.G., on behalf of the Early Breast Cancer Trialist's Collaborative Group: Effect of obesity in premenopausal ER+ early breast cancer: EBCTCG data on 80,0000 patients in 70 trials. ASCO Annual Meeting. Abstract 503. Presented May 31, 2014.

25. Eggermont A.M., Chiarion-Sileni V., Grob J.J., Dummer R., Wolchok J.D., Schmidt H., Hamid O., Robert C., Ascierto P.A., Richards J.M., Lebbé C., Ferraresi V., Smylie M., Weber J.S., Maio M., Bastholt L., Mortier L., Thomas L., Tabir S., Hauschild A., Hassel J.C., Hodi F.S., Taitt C., de Pril V., de Schaetzen G., Suciu S., Testori A. Prolonged Survival in Stage III Melanoma with Ipilimumab Adjuvant Therapy. N Engl J Med. 2016 Oct 7. [Epub ahead of print]. doi: 10.1056/ NEJMoa1611299. PMID: 27717298.

26. Moore M.J., Goldstein D., Hamm J., Figer A., Hecht J.R., Gallinger S., Au H.J., Murawa P., Walde D., Wolff R.A., Campos D., Lim R., Ding K., Clark G., Voskoglou-Nomikos T., Ptasynski M., Parulekar W.; National Cancer Institute of Canada Clinical Trials Group. Erlotinib plus gemcitabine compared with gemcitabine alone in patients with advanced pancreatic cancer: a phase III trial of the National Cancer Institute of Canada Clinical Trials Group. J Clin Oncol. 2007 May 20; 25(15): 1960-6. doi: 10.1200/JCO.2006.07.9525. PMID: 17452677. 